\title{
Impact of Sepsis Intervention Protocol (SIP) on Adherence to Three- hour and Six-hour Bundles and Mortality Outcomes in the Emergency Department
}

Osagie Igiebor, Mohamed Nakeshbandi, Ninfa Mehta, Randi Ozaki, Michael Lucchesi, Maryanne Daley, Moro O. Salifu and Samy I. McFarlane*

Departments of Internal Medicine and Emergency Medicine, University Hospital of Brooklyn SUNY- Downstate Health Science University, Brooklyn, NY, USA

\section{Abstract}

Sepsis is a commonly encountered diagnosis affecting nearly 1.7 million adults in the USA annually. According to Center for Disease Control (CDC), over 270,000 Americans die of sepsis each year and 1 in 3 hospital mortalities is attributed to sepsis.

The Surviving Sepsis Campaign (SSC) Guidelines for management of severe sepsis and septic shock published in 2004 provide key elements in the treatment of sepsis that are organized into two bundles of care, the "resuscitation" and "management" bundles, including interventions to be accomplished within specified timeframes. In this quality improvement study, we implemented a sepsis intervention protocol (SIP) intended to increase adherence to 3-hour and 6-hour bundles, and to examine the impact on mortality of patients presenting with severe sepsis and septic shock in our emergency department.

We analyzed data from our emergency department as reported to the New York State Department of Health (NYSDOH) from 2017Q2 to 2018Q2 (April 2017 -June 2018), the period prior to implementation of SIP, compared to data from 2018Q3 to 2019Q2 (July 2018 to June 2019) after implementation of SIP. The implementation of SIP resulted in increased3-hour and 6-hour bundle adherence and showed a clinically significant reduction of the mean pre-intervention hospital percent mortality of $40.3 \%$ to a mean post-intervention hospital percent mortality of $28.7 \%$. A t-test analysis of the pre and post intervention mean hospital percent mortality revealed a reduction in mortality outcomes that was also statistically significant $(\mathrm{p}<0.05)$. Our study demonstrates that a well-designed and implemented SIP can increase bundle adherence and is highly effective in reducing mortality among high-risk population.

\section{Publication History:}

Received: May 08, 2020

Accepted: July 18, 2020

Published: July 20, 2020

\section{Keywords:}

COVID-19, Pathogenesis, SARSCoV-2, Acute Kidney Injury, Cardiac manifestation, Multiorgan failure

\section{Introduction}

Sepsis is a major healthcare problem, with high incidence and poor mortality outcome [1]. The Surviving Sepsis Campaign (SSC) was first launched in 2002 with the goals of increasing clinician and public awareness of the sepsis problem, enhancing the standard of care and decreasing mortality [2]. The SSC Guidelines for management of severe sepsis and septic shock were first published in 2004 [3], providing recommendations that are intended to guide clinical practice. In order to facilitate the implementation of the guidelines, the key elements in the treatment of sepsis were organized into two bundles of care, the "resuscitation" and "management" bundle, including interventions to be accomplished within specified timeframes [4]. A bundle is a set of diagnostic or therapeutic processes that when implemented as a group may act synergistically, providing a greater survival benefit than each individual component.

Several published data show that performance improvement or implementation programs on compliance with sepsis bundles was associated with lower risk of death in patients with severe sepsis and septic shock [5,6]. Thus, On May 1, 2013, NYSDOH Title $10 \mathrm{New}$ York Codes Rules and Regulations (NYCRR) Sections 405.2 and 405.4 were amended to require that hospitals have in place evidence-based protocols for the early recognition and treatment of patients with severe sepsis and septic shock that are based on generally accepted standards of care. Medical staffs were responsible for the collection, use, and reporting of quality measures related to the recognition and treatment of severe sepsis for purposes of internal quality improvement and hospital reporting to the department.
However, consistent adherence to sepsis bundles in the management of patients has remained a challenge. The purpose of this quality improvement project was to examine the outcome implications of implementing a sepsis intervention protocol to increase adherence to 3-hour and 6-hour bundle, and the impact on mortality in our emergency department.

\section{Methods}

\section{Design, overview and setting}

This quality improvement study was performed in the emergency department. Our study protocol was reviewed and approved by our Institutional Review Board (IRB) with a waiver of informed consent as the study was deemed a performance improvement intervention.

\section{Patients}

All adult patients who presented to the emergency department from September 2018 through June 2019, were screened for this "Corresponding Author: Prof. Samy I. McFarlane, College of Medicine, Department of Medicine, Division of Endocrinology, Internal Medicine Residency Program Director, State University of New York, Downstate Medical Center, 450 Clarkson Ave, Box 50, Brooklyn, New York 11203-2098, USA. Phone 718-2706707, Fax 718-270-4488; E-mail: smcfarlane@downstate.edu

Citation: Igiebor O, Nakeshbandi M, Mehta N, Ozaki R, Lucchesi M, et al. (2020) Impact of Sepsis Intervention Protocol (SIP) on Adherence to Three-hour and Sixhour Bundles and Mortality Outcomes in the Emergency Department. Int J Clin Res Trials 5: 149. doi: https://doi.org/10.15344/2456-8007/2020/149

Copyright: (c) 2020 Igiebor et al. This is an open-access article distributed under the terms of the Creative Commons Attribution License, which permits unrestricted use, distribution, and reproduction in any medium, provided the original author and source are credited. 
Citation: Igiebor O, Nakeshbandi M, Mehta N, Ozaki R, Lucchesi M, et al. (2020) Impact of Sepsis Intervention Protocol (SIP) on Adherence to Three-hour and Six-hour Bundles and Mortality Outcomes in the Emergency Department. Int J Clin Res Trials 5: 149. doi: https://doi.org/10.15344/2456-8007/2020/149

Page 2 of 8

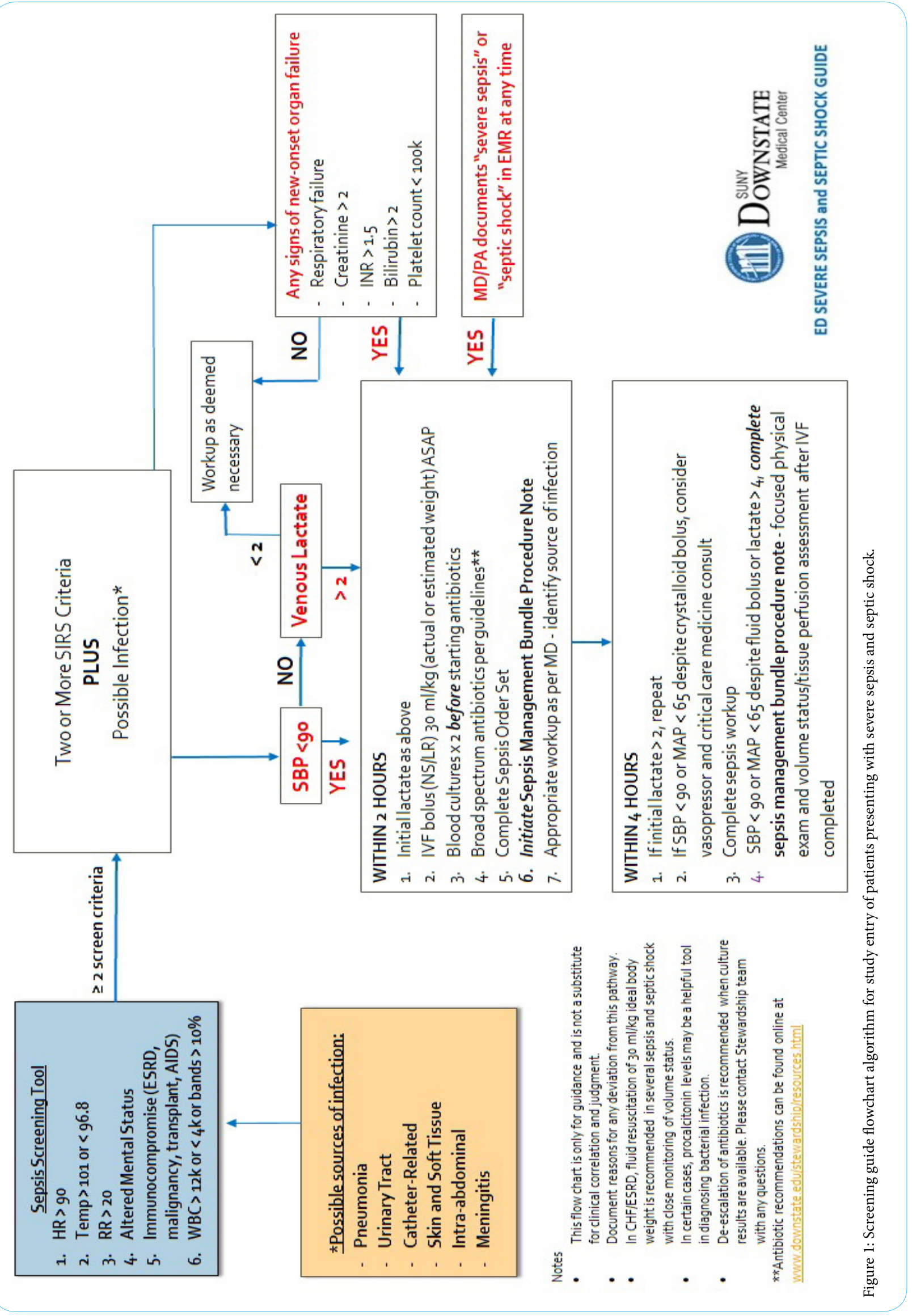


Citation: Igiebor O, Nakeshbandi M, Mehta N, Ozaki R, Lucchesi M, et al. (2020) Impact of Sepsis Intervention Protocol (SIP) on Adherence to Three-hour and Six-hour Bundles and Mortality Outcomes in the Emergency Department. Int J Clin Res Trials 5: 149. doi: https://doi.org/10.15344/2456-8007/2020/149

Page 3 of 8

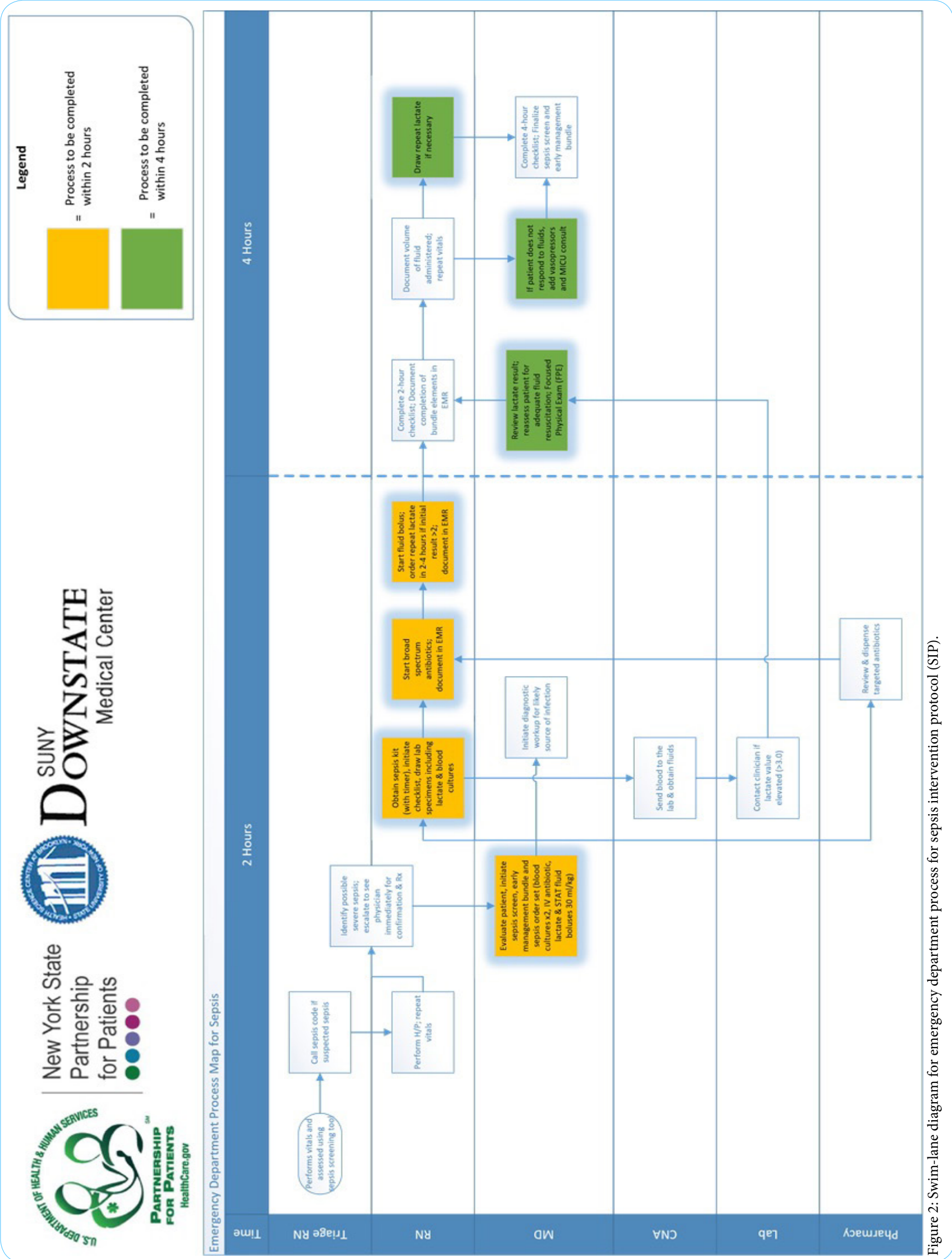


Citation: Igiebor O, Nakeshbandi M, Mehta N, Ozaki R, Lucchesi M, et al. (2020) Impact of Sepsis Intervention Protocol (SIP) on Adherence to Three-hour and Six-hour Bundles and Mortality Outcomes in the Emergency Department. Int J Clin Res Trials 5: 149. doi: https://doi.org/10.15344/2456-8007/2020/149

Page 4 of 8

\section{(iii) $\mathrm{D}^{\operatorname{sun}}$ OWNSTATE Medical Center}

1. Blood Cultures drawn within 2 hours of SS/shock, prior to antibiotics

Exceptions:

$\square$ Patient/Family refused

$\square$ Difficult vascular access

$\square$ other:

2. Antibiotics were administrated within 2 hours of SS/shock

Exceptions:

$\square$ Patient/Family refused

$\square$ Difficult vascular access

口other:

3. Initial lactate was collected STAT for SS/shock

$\square$ Yes $\square$ No

Exceptions: $\square$ Patient/Family refused $\square$ Difficult vascular access

口other:

5. IV fluids (NS or LR) were started at $30 \mathrm{ml} / \mathrm{kg}=\ldots$ within 2 hours of $\mathrm{SS} / \mathrm{shock} \square$ Yes $\square$ No

(PEDS: $20 \mathrm{ml} / \mathrm{kg}=$

Exceptions: USE IDEAL BODY WEIGHT FOR BMI>30, VOLUME OVERLOAD, PULMONARY EDEMA

$\square$ Patient/Family refused $\quad \square$ Difficult vascular access

$\square \mathrm{MD} / \mathrm{PA}$ determined fluids not clinically appropriate due to:

6. Repeat lactate drawn within 2 hours of SS/shock

$\square$ Yes $\square$ No

Exceptions:

7. Patient responsive to fluid resuscitation:

$\square$ Yes $\square$ No

8. If not responsive to fluids, vasopressor started

(only if given): $\square$ Yes $\square$ No

9. Focused Physical Exam documented/attested to by MD/PA within 4 hours: $\square$ Yes $\square$ No

After IVFs completed. 5 of 8 elements below.

$\square$ vS $\times 4 \square$ CP exam $\square$ Cap refill $\square$ Periph pulse $\square$ skin exam $\square 02$ sat $\square$ Urine Output $\square$ sI (Shock index)

RN Signature:

ED Review:

Comments:
Physician/Pa Signature:

PI Coordinator:

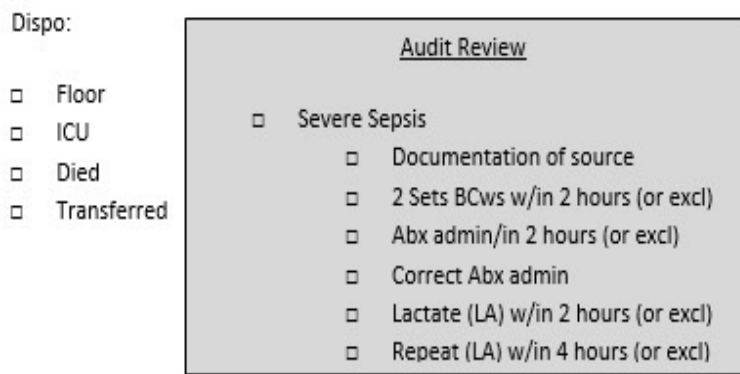

\section{Audit Review}

- Septic Shock

a Met elements of severe sepsis

- IVF begun w/in 2 hrs (or excl)

口 $30 \mathrm{ml} / \mathrm{kg} \mathrm{IVF} \mathrm{w/in} 2$ hrs (or excl)

- Vasopressor begun $\mathrm{w} / \mathrm{in} 4 \mathrm{hrs}$

a Repeat Focused PE W/in 4 hrs

Figure 3: Sepsis checklist for implementation of time sensitive measures in the sepsis resuscitation and management bundles. 
Citation: Igiebor O, Nakeshbandi M, Mehta N, Ozaki R, Lucchesi M, et al. (2020) Impact of Sepsis Intervention Protocol (SIP) on Adherence to Three-hour and Six-hour Bundles and Mortality Outcomes in the Emergency Department. Int J Clin Res Trials 5: 149. doi: https://doi.org/10.15344/2456-8007/2020/149

Page 5 of 8

project. Patients with severe sepsis or septic shock at the time of presentation as defined by Center for Medicare and Medicaid service, were identified through the use of a written screening guide (Figure 1).

\section{Sepsis intervention protocol}

ED physicians, nurses, CNA, pharmacists and lab technician were educated regarding implementation of time sensitive measures of sepsis bundles through lectures and monthly nursing orientation at the end of September through October of 2018 (Figure 2). Sepsis posters highlighting time sensitive measures were posted throughout the emergency department. Time sensitive measures in the 3-hour bundle include obtaining timely initial lactate level, administration of IV fluids at a rate of $30 \mathrm{ml} / \mathrm{kg}$ of body weight within 2 hours, and timely blood cultures draw prior to administration of antibiotics. In the 6-hour bundle, time sensitive measures include obtaining repeat lactate within 4 hours if initial lactate $>2$, assessing patient responsiveness to fluid resuscitation and prompt administration of vasopressors within 4 hours if patient is not responsive to fluid resuscitation.

An ED sepsis kit which included two liters of crystalloids, and a timer as reminder for timely re-measurement of lactate was created. Patients presenting to the ED who demonstrated clinical deterioration and met two or more criteria on the sepsis screening guide (thereby demonstrating that they were likely to have severe sepsis or septic shock) were intended to trigger a sepsis code (SIP=sepsis intervention protocol). Sepsis resuscitation and management bundle elements were implemented using a sepsis checklist to capture the above time sensitive measures to be completed by nurses and physicians (Figure 3). Checklist review process by Quality Assurance and Performance Improvement department along with ED leadership allowed for real time feedback.

\section{Results}

We analyzed data of cases of patients with severe sepsis and septic shock in our emergency department which were reported to the New York State Department of Health (NYSDOH) from 2017 Q2 to 2018 Q2 (April 2017 - June 2018), the period prior to implementation of SIP. We compared the results to data from 2018 Q3 to 2019 Q2 (July 2018 to June 2019) the period after implementation of SIP. A total of 512 cases of patient with severe sepsis and septic shock was reported, of which there were a total of 180 deaths during the time under review for this project.

A demographic analysis of hospital data pre and post implementation of SIP showed a higher proportion of patient aged 70 to 79 years (about 24 to $26 \%$ of total number of cases) constituting the majority of patients with severe sepsis or septic shock. Of note, 88.4 to 88.6 $\%$ of total number of cases in our hospital were African Americans compared to statewide averages of which only 15.9 to $16.3 \%$ were African Americans. A mild gender difference was also noted with female patient constituting a slightly higher majority (of 50.2 to $53.6 \%$ ) compared to (46.4 to $49.8 \%$ ) of male cases in our hospital. The reverse situation was noted when comparing statewide data which showed a slightly higher male to female percentages (Table 1a \& 1b).

As show in the trend percentages in figure 4, prior to 2018 Q3 our hospital percentages of timely lactate level, timely blood culture prior to antibiotic administration and timely re-measurement of lactate level in sepsis cases, were below statewide percentages. Consequently, 3-hour and 6-hour bundle adherence percentages were also below statewide averages (Figure 4e \& 4f). Of note however, timely broadspectrum antibiotic administration was similar or above statewide percentages.

\begin{tabular}{|c|c|c|c|}
\hline & \multicolumn{2}{|c|}{ Your Hospital } & \multirow{2}{*}{$\begin{array}{r}\text { State } \\
\%\end{array}$} \\
\hline & $\mathrm{N}$ & $\%$ & \\
\hline Total & 211 & & \\
\hline \multicolumn{4}{|l|}{ Age } \\
\hline $18-29$ & 7 & $3.33 \%$ & $2.7 \%$ \\
\hline $30-39$ & 0 & $0.0 \%$ & $3.6 \%$ \\
\hline $40-49$ & 15 & $7.1 \%$ & $6.1 \%$ \\
\hline $50-59$ & 25 & $11.8 \%$ & $13.3 \%$ \\
\hline $60-69$ & 50 & $23.7 \%$ & $20.9 \%$ \\
\hline $70-79$ & 56 & $26.5 \%$ & $23.4 \%$ \\
\hline $80-89$ & 40 & $19.0 \%$ & $21.0 \%$ \\
\hline $90-110$ & 18 & $8.5 \%$ & $9.0 \%$ \\
\hline \multicolumn{4}{|l|}{ Gender } \\
\hline Male & 98 & $46.4 \%$ & $51.8 \%$ \\
\hline Female & 113 & $53.6 \%$ & $48.1 \%$ \\
\hline Unknown & 0 & $0.0 \%$ & $0.0 \%$ \\
\hline \multicolumn{4}{|l|}{ Race Group } \\
\hline White & 10 & $4.7 \%$ & $64.1 \%$ \\
\hline African American & 187 & $88.6 \%$ & $16.3 \%$ \\
\hline Native American & 0 & $0.0 \%$ & $0.2 \%$ \\
\hline Asian & 5 & $2.4 \%$ & $4.0 \%$ \\
\hline Pacific Islander & 0 & $0.0 \%$ & $0.1 \%$ \\
\hline Other Race & 9 & $4.3 \%$ & $14.1 \%$ \\
\hline Multi-racial & 0 & $0.0 \%$ & $1.2 \%$ \\
\hline \multicolumn{4}{|l|}{ Ethnicity } \\
\hline Spanish/Hispanic Origin & 14 & $6.6 \%$ & $11.2 \%$ \\
\hline Not of Spanish/Hispanic Origin & 190 & $90.0 \%$ & $79.8 \%$ \\
\hline Unknown & 7 & $3.3 \%$ & $9.0 \%$ \\
\hline
\end{tabular}

Table 1a: Cumulative demographic distribution data of patients from 2017 Q3 to 2018 Q2 prior to implementation of SIP.

\begin{tabular}{|c|c|c|c|}
\hline & You & ospital & State \\
\hline & $\mathrm{N}$ & $\%$ & $\%$ \\
\hline Total & 225 & & \\
\hline Age & & & \\
\hline $18-29$ & 9 & $4.0 \%$ & $2.6 \%$ \\
\hline $30-39$ & 17 & $7.6 \%$ & $3.6 \%$ \\
\hline $40-49$ & 18 & $8.0 \%$ & $5.7 \%$ \\
\hline $50-59$ & 38 & $16.9 \%$ & $13.6 \%$ \\
\hline $60-69$ & 41 & $18.2 \%$ & $21.7 \%$ \\
\hline $70-79$ & 54 & $24.0 \%$ & $23.5 \%$ \\
\hline $80-89$ & 31 & $13.8 \%$ & $20.6 \%$ \\
\hline $90-110$ & 17 & $7.6 \%$ & $8.7 \%$ \\
\hline Gender & & & \\
\hline Male & 112 & $49.8 \%$ & $53.4 \%$ \\
\hline Female & 113 & $50.2 \%$ & $46.5 \%$ \\
\hline Unknown & 0 & $0.0 \%$ & $0.1 \%$ \\
\hline Race Group & & & \\
\hline White & 15 & $6.7 \%$ & $63.8 \%$ \\
\hline African American & 119 & $88.4 \%$ & $15.9 \%$ \\
\hline Native American & 1 & $0.4 \%$ & $0.2 \%$ \\
\hline Asian & 4 & $1.8 \%$ & $4.1 \%$ \\
\hline Pacific Islander & 0 & $0.0 \%$ & $0.0 \%$ \\
\hline Other Race & 5 & $2.2 \%$ & $14.5 \%$ \\
\hline Multi-racial & 1 & $0.4 \%$ & $1.4 \%$ \\
\hline Ethnicity & & & \\
\hline Spanish/Hispanic Origin & 15 & $6.7 \%$ & $10.7 \%$ \\
\hline Not of Spanish/Hispanic Origin & 205 & $91.1 \%$ & $81.4 \%$ \\
\hline Unknown & 5 & $2.2 \%$ & $7.8 \%$ \\
\hline
\end{tabular}

Table 1b: Cumulative demographic distribution of patients from 2018 Q3 to 2019 Q2 post implementation of SIP. 
Citation: Igiebor O, Nakeshbandi M, Mehta N, Ozaki R, Lucchesi M, et al. (2020) Impact of Sepsis Intervention Protocol (SIP) on Adherence to Three-hour and Six-hour Bundles and Mortality Outcomes in the Emergency Department. Int J Clin Res Trials 5: 149. doi: https://doi.org/10.15344/2456-8007/2020/149

Page 6 of 8

The implementation of SIP in 2018 Q3, resulted in improved adherence and performance of the sepsis bundle (Figure 4). Consequently, a clinically significant reduction of the mean preintervention hospital percent mortality of $40.3 \%$ to a mean postintervention hospital percent mortality of $28.7 \%$ was observed (Figure 5). Furthermore, as shown in Table 2, a two-sample t-test analysis of the mean hospital percent mortality before and after implementation of SIP demonstrated a reduction in mortality outcome that was statistically significant $(p<0.05)$.

\section{Discussion}

This quality improvement project demonstrates that an evidencebased protocol for the early recognition and treatment of patients with severe sepsis and septic shock is an effective tool for increasing

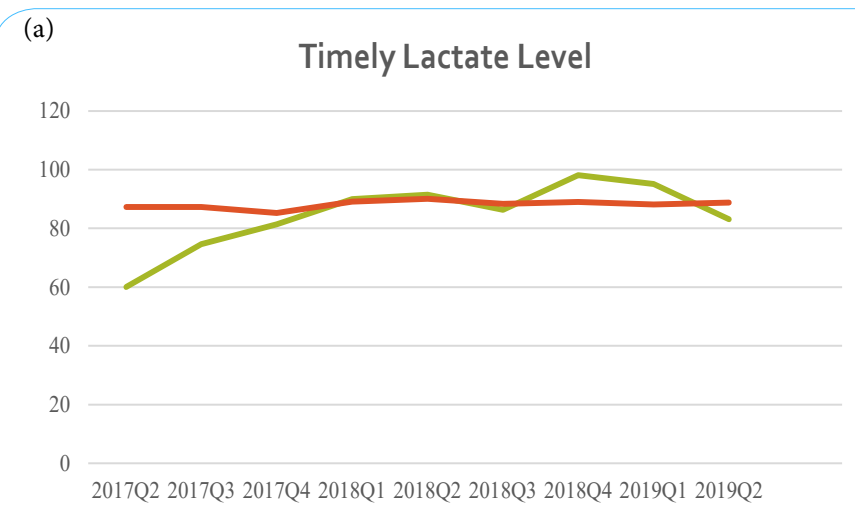

(c)

$$
\text { Hospital\% }
$$

\section{Timely Broad Spectum Antibiotics administration}

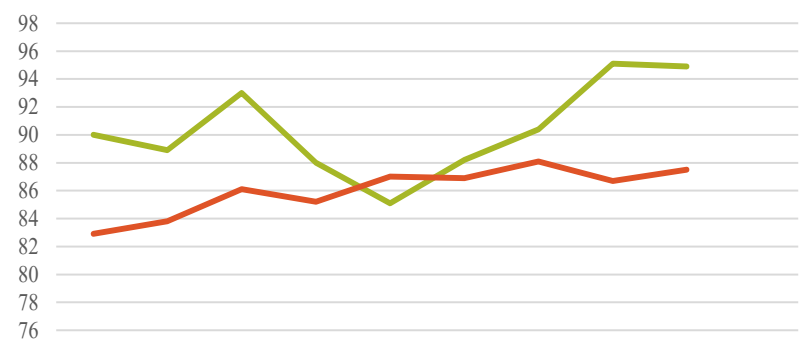

2017Q2 2017Q3 2017Q4 2018Q1 2018Q2 2018Q32018Q4 2019Q12019Q2

Hospital\% —

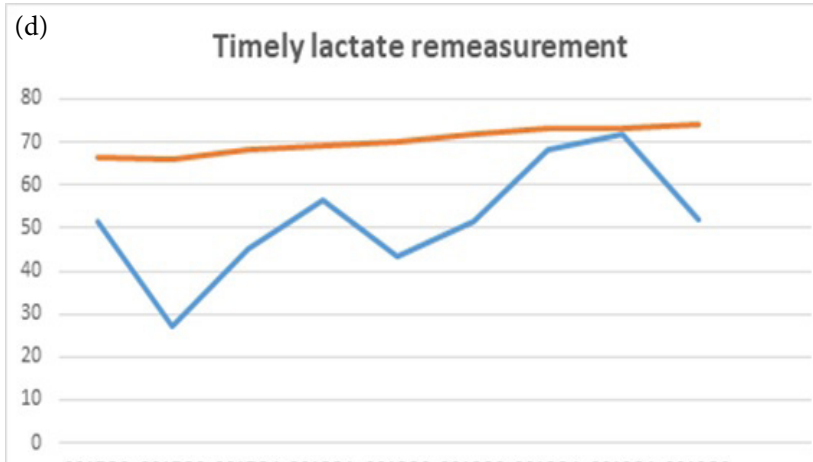

$2017 Q 22017 Q 32017 Q 42018 Q 12018 Q 22018 Q 32018 Q 42019 Q 12019 Q 2$

$$
\text { -Hospital\% _tatewide\% }
$$

compliance with sepsis resuscitation and management bundle elements as its primary outcome. The use of a sepsis checklist by health care providers after the implementation of SIP in October 2018 significantly improved performance bundle elements. The most remarkable finding in this project was the statistically significant reduction in mortality outcomes of patient with severe sepsis and septic shock since implementation of the SIP.

We did not perform a statistical analysis of each bundle elements to assess if the improved trended percent performance of these elements upon implementation of SIP were statistically significant. Furthermore, we noted that timely broad spectrum antibiotic administration were also above or similar to statewide percentages before and after implementation of SIP. The timeliness of antibiotic administration has been proven to decrease mortality. Thus, reducing

(b)

Timely Blood Culture prior to antibiotics

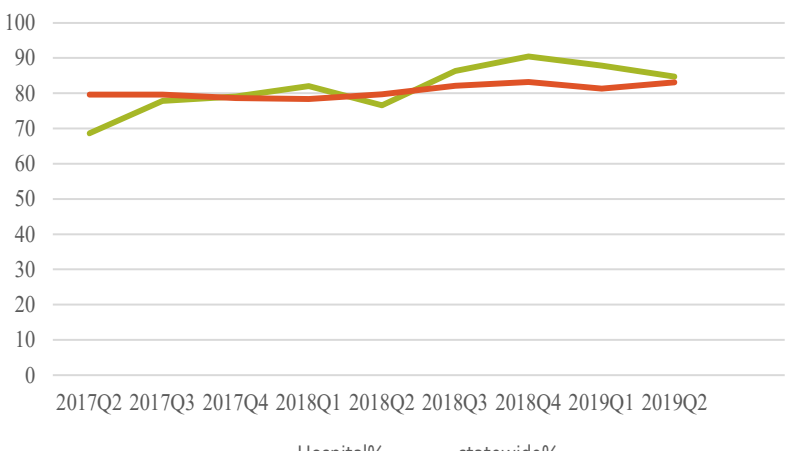

(d)

Trended 3-hour bundle adherence percentage by quarter, compared to statewide

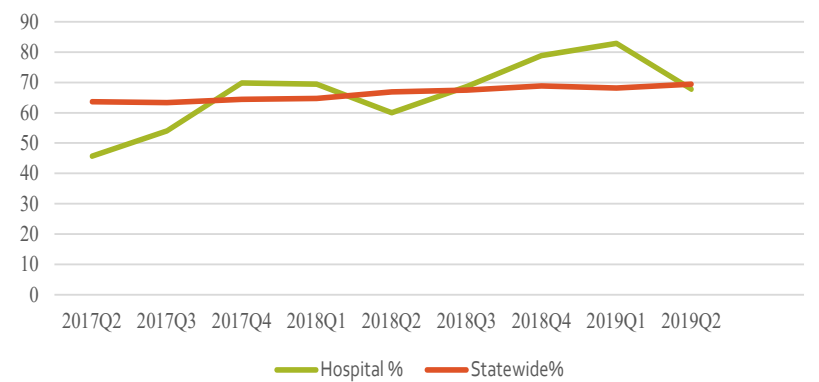

(f) Trended 6-hour bundle adherence percentage by quarter, compared to statewide

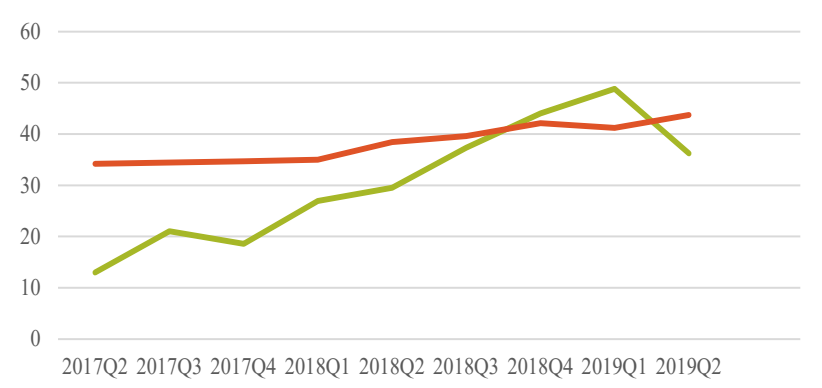

hospital \%

Figure 4: Trended hospital percentages compared to statewide percentages of: (a) timely lactate level, (b) timely blood culture prior to antibiotics (c) timely broad spectrum antibiotic administration (d) timely lactate re-measurement (e) 3-hour bundle adherence and (f) 6-hour bundle adherence. 
Citation: Igiebor O, Nakeshbandi M, Mehta N, Ozaki R, Lucchesi M, et al. (2020) Impact of Sepsis Intervention Protocol (SIP) on Adherence to Three-hour and Six-hour Bundles and Mortality Outcomes in the Emergency Department. Int J Clin Res Trials 5: 149. doi: https://doi.org/10.15344/2456-8007/2020/149

\section{Trended mortality percentages by quarter, compared to statewide}

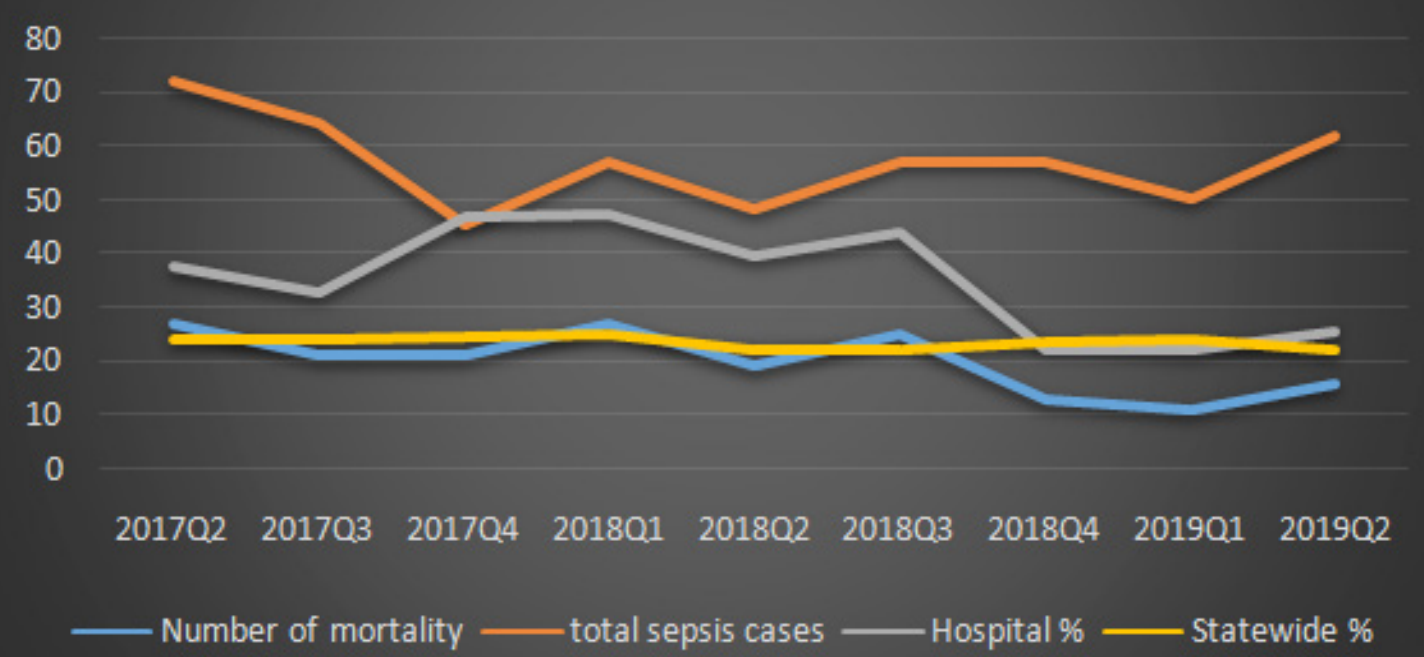

Figure 5: Trended mortality percentages by quarter, compared to statewide.

\begin{tabular}{|l|l|l|}
\hline \multicolumn{3}{|c|}{ t-Test: Two-Sample Assuming Unequal Variances } \\
\hline & Pre-intervention & Post-intervention \\
\hline Mean & $\mathbf{4 0 . 8}$ & $\mathbf{2 8 . 7 2 5}$ \\
\hline Variance & 38.67 & 104.86 \\
\hline Observations & 5 & 4 \\
\hline t Stat & $\mathbf{2 . 0 7}$ & \\
\hline $\mathbf{P}(\mathbf{T}<=$ t) one-tail & $\mathbf{0 . 0 4}$ & \\
\hline
\end{tabular}

Table 2: Two-Sample t-test of mean hospital percent mortality pre and post implementation of SIP.

antimicrobial burden by early antibiotic administration not only decreases mortality in septic shock, but also decreases pressor/ inotrope free days, and ventilator days [7-11]. However, the findings of this quality improvement project demonstrates that improved performance of bundle elements in addition to prompt antibiotics administration results in decrease mortality outcomes.

For example, elevated lactate results from hypoperfusion of tissues. Decreased perfusion to tissue affects mitochondrial oxidative phosphorylation thereby shifting energy metabolism to anaerobic glycolysis and the production of serum lactic acid [6]. Animal models have shown that a delay in antibiotic administration following the onset of hypotension and hypoperfusion of tissues is associated with an increase in inflammatory mediators [12]. Therefore, timely measurement of lactate levels may alert providers to perfusion abnormalities that may prompt therapeutic changes such as prompt intravenous fluid and broad spectrum antibiotic administration.

\section{Conclusion}

In conclusion, early intervention protocols for medical emergencies with an extremely high mortality rate such as Code STEMI for patients with ST-segment elevation myocardial infarctions have demonstrated an improved rate of survival with early recognition and implementation of appropriate therapy. Severe sepsis and septic shock are medical emergencies with an extremely high mortality rate. A sepsis intervention protocol (SIP) as demonstrated in this project can be useful in severe sepsis and septic shock as it increases completion of time-sensitive measures via the use of a sepsis checklist, compliance with bundle elements and improves mortality outcomes. The SIP was easy to implement, essentially without cost, improved compliance with sepsis bundle and mortality outcomes, making it an attractive tool for institutions to consider when meeting the goals of early goaldirected therapy for severe sepsis and septic shock.

\section{Competing Interests}

The authors declare that they have no competing interests.

\section{Acknowledgement}

This work is supported, in part, by the efforts of Dr. Moro O. Salifu M.D., M.P.H., M.B.A., M.A.C.P., Professor and Chairman of Medicine through NIH Grant number S21MD012474.

\section{References}

1. Mayr FB, Yende S, Angus DC (2014) Epidemiology of severe sepsis. Virulence 5: 4-11.

2. Townsend SR, Schorr C, Levy MM, Dellinger RP (2008) Reducing mortality in severe sepsis: The Surviving Sepsis Campaign. Clin Chest Med 29: 721-733.

3. Dellinger RP, Levy MM, Rhodes A, Annane D, Gerlach H, et al. (2013) Surviving Sepsis Campaign: International Guidelines for the Management of Severe Sepsis and Septic Shock: 2012. Crit Care Med 41: 580-637.

4. Levy MM, Pronovost PJ, Dellinger RP, Townsend S, Resar RK, et al. (2004) Sepsis change bundles: Converting guidelines into meaningful change in behavior and clinical outcome. Crit Care Med 32: 595-597.

5. Gao F, Melody T, Daniels DF, Giles S, Fox S, et al. (2005) The impact of compliance with 6-hour and 24-hour sepsis bundles on hospital-mortality in patients with severe sepsis: a prospective observational study. Crit Care 9: $764-770$.

6. Jones AE, Shapiro NI, Trzeciak S, Arnold RC, Claremont HA, et al. (2010) Lactate clearance vs central venous oxygen saturation as goals of early sepsis therapy: a randomized clinical trial. JAMA 303: 739-746. 
Citation: Igiebor O, Nakeshbandi M, Mehta N, Ozaki R, Lucchesi M, et al. (2020) Impact of Sepsis Intervention Protocol (SIP) on Adherence to Three-hour and Six-hour Bundles and Mortality Outcomes in the Emergency Department. Int J Clin Res Trials 5: 149. doi: https://doi.org/10.15344/2456-8007/2020/149

Page 8 of 8

7. Kumar A, Zarychanski R, Light B, Parrillo J, Maki D, et al. (2010) Early combination antibiotic therapy yields improved survival compared with monotherapy in septic shock: a propensity-matched analysis. Critical Care Medicine 38: 1773-1785.

8. Kumar A, Ellis P, Arabi $Y$, Roberts D, Light $B$, et al. (2009) Initiation of inappropriate antimicrobial therapy results in a fivefold reduction of survival in human septic shock. Chest 136: 1237-1248.

9. Garnacho-Montero J, Garcia-Garmendia JL, Barrero-Almodovar A, JimenezJimenez FJ, Perez-Paredes C, et al. (2003) Impact of adequate empirical antibiotic therapy on the outcome of patients admitted to the intensive care unit with sepsis. Critical Care Medicine 31: 2742-2751.

10. Ibrahim EH, Sherman G, Ward S, Fraser VJ, Kollef MH, et al. (2000) The influence of inadequate antimicrobial treatment of bloodstream infections on patient outcomes in the ICU setting. Chest 118: 146-155.

11. Harbarth S, Garbino J, Pugin J (2000) Inappropriate initial antimicrobial treatment of bloodstream infections on patient outcomes in the ICU setting. Chest 118: 9-11.

12. Watts JA, Kline JA (2003) Bench to bedside: the role of mitochondrial medicine in the pathogenesis and treatment of cellular injury. Academic Emergency Medicine 10: 985-997. 